\title{
Le sfide pastorali dopo le Giornate Mondiali della Gioventù 2016
}

\section{Pastoral Challenges After World Youth Day 2016}

\begin{abstract}
World Youth Day (WYD) 2016 gathered a powerful body of youth, who are now asking; What's next? Without a doubt this group of young people is the promising group within the Church. They are brave and consciously living their faith. They have lived something that has radically transformed them. There are not many of them, yet they are strong. They are excelling and forming a wedge in a growing secular environment. What about those who are left? What's happening with them? Who are today's Polish youth and what dilemmas are they facing? As the results of a study completed on the topic of youth and their spirituality shows, we can generally argue that the currently growing generation is a consumerist one. We are also experiencing a growing discrepancy between declared faith, active practice of faith, and morality. Today's youth, essentially, are not concerned with matters of faith and carry a negative image of the congregation and priests. What indications may be implemented in the ministry of our youth after such a great event as the World Youth Day? What is the ministry of youth and how can we realize it?
\end{abstract}

\section{Keywords}

Youth, ministry of youth, Pope Francis, young generation, today's youth, ministry challenges, World Youth Day (WYD) 2016, a consumerist generation, language of preaching. 


\section{Il messaggio del Papa ai giovani}

Non intendo analizzare qui le omelie del Papa. Vorrei solo prendere da esse ciò che potrebbe essere utile per la pastorale della gioventù.

Ci sono due elementi fondamentali: quello che il Papa ha detto e come lo ha detto. La cosa più importante che lui ha ricordato ai giovani è stata quella che esistono due mondi: il mondo terreno e quello spirituale. La realtà terrena potrebbe nascondere ai giovani il mondo di Dio. Secondo il Papa, il dovere della Chiesa sta nel rivelare ai giovani il mondo spirituale con lo scopo di portarli ad incontrare Gesù.

Perché questo è così importante? Perché l'incontro con Gesù sconvolge tutto.

Quindi in che consiste oggi la pastorale giovanile? Consiste proprio nello scoprire il mondo spirituale impolverato di temporalità. Consiste anche nell'assistere all'incontro di un cuore umano con Dio. Così poco e così tanto. "Quando Gesù tocca il cuore dell'uomo, quest'uomo diventa capace di fare opere grandiose», dice Papa Francesco.

Come esercitare una tale pastorale? Dall'intero messaggio del Papa spunta una sorta di metodologia. Nelle sue omelie il Papa non impone nulla. Il suo metodo consiste piuttosto nel dare dritte, suggerimenti, consigli e indicazioni. Egli parlava motivando e suscitando riflessioni. Voleva trattenere i giovani affinché essi potessero rifletterci su. Nel suo predicare non c'era niente di moraleggiante. Come prima cosa rivelava agli ascoltatori una visione di Dio: 'Egli comeè. Soltanto dal fascino sulla bontà e sulla misericordia di Dio possono nascere atteggiamenti morali. Mostrava quello che è bello e quello che risulta solo un'apparenza della bellezza. "Che volete scegliere?» - chiedeva. Non sceglieva per loro. Conduceva una propria riflessione per suscitare nel giovane uomo un dialogo interno.

Volevo far presente tutto questo perché conviene prendere in considerazione tale metodo nella pastorale della gioventù. I giovani dicono apertamente e senza mezzi termini: «La Chiesa non può impormi nulla! Ho il mio cervello e so quello che voglio». I giovani impazziscono per tutto ciò che riguarda la libertà. Non possiamo sentirci offesi per un tale atteggiamento, ma dobbiamo invece cercare una chiave. La chiave per il cuore dei giovani sará sicuramente il linguaggio della predicazione. Papa Francesco ha parlato loro in un modo semplice, pronunciava delle frasi-chiave brevissime. Predicava il Vangelo nel loro linguaggio. Le espressioni quali «disco rigido - la memoria di Dio», «il Vangelo quale navigatore sulle strade della vita», «la droga del pensare», «installare una 
connessione stabile con Dio», «la chat evangelica», tutto questo è davvero il linguaggio quotidiano dei giovani. Loro lo ascoltavano, sapevano di che cosa Egli stava parlando. Non si trattava però delle prediche prodotte dal computer, ma di prediche derivanti dal cuore e trasmesse con un linguaggio utilizzato nella comunicazione via computer. Il Dio sperimentato da Francesco e comunicato agli ascoltatori nel loro proprio linguaggio, Francesco che ci invita a soffermarci, convincente e a volte commovente.

Che cosa ha detto loro il Papa? Ha detto di esser venuto per aiutarli. Non accusava né ammoniva. Individuava gli aspetti più deboli della condizione dei giovani e mostrava come gestire la vita per renderla significativa e bella. Quanti giovani non credono in sé, non credono di essere bravi. Proprio a questi giovani il Papa è venuto a dare una mano. «Non accettarsi, vivere scontenti e pensare in negativo significa non riconoscere la nostra identità più vera (...) Dio ci ama così come siamo e nessun peccato, difetto o sbaglio Gli farà cambiare idea (...) Lo sguardo di Gesù va oltre i difetti e vede la persona; non si ferma al male del passato, ma intravvede il bene nel futuro». ${ }^{1}$

Così Papa Francesco ha aperto i nuovi orizzonti ai giovani, internamente confusi. Inoltre nel suo messaggio ha risvegliato i desideri dormienti e i sogni dei giovani. Ha rafforzato quel bene che cè in loro. $\mathrm{E}$ a volte lo ha fatto in un modo provocatorio, dicendo loro: «Giovani pensionati, volete essere così? (...) Annoiati e noiosi, che annoiano gli altri. (...) Giovani pensionati che 'gettano la spugna' prima di iniziare la partita (...) Giovani-divano (...) Volete essere addormentati, imbambolati e intontiti? (...) è più facile e vantaggioso avere dei giovani così che avere giovani svegli, desiderosi di rispondere al sogno di Dio e a tutte le aspirazioni del cuore». ${ }^{2}$ Questo modo provocatorio di parlare potrà lasciare i giovani beati e tranquilli? Questo dialogo è stato anche accompagnato da un invito ad essere responsabili non solo per se stessi, ma per il mondo, per l'umanità. Il Papa ha detto: «l'umanità necessita oggi di gente che non vuole vivere a metà». ${ }^{3}$ Ha incoraggiato i giovani a fare della propria vita un dono e lo ha chiamato 'eroismo nel servire'. Se non viviamo per donarci al servizio, neghiamo Gesù Cristo. Riferendosi ad un mondo inquieto ha incoraggiato tutti noi a diventare una famiglia e ha indicato le tre caratteristiche di questa famiglia: fratellanza, amicizia e unità.

1 Franciszek Ojciec Święty, Błogosławieni miłosierni. Słowa do młodych, Kraków 2016, pp. 61-68.

${ }^{2}$ Franciszek Ojciec Święty, Błogosławieni miłosierni, pp. 100-106.

${ }^{3}$ Franciszek Ojciec Święty, Błogosławieni miłosierni, pp. 89-98. 
Ancora due cose risultano importanti per una pastorale giovanile. Molto spesso consideriamo i giovani come il gruppo sociale più importante. Probabilmente è giusto dato che i giovani sono il futuro di ogni nazione. Tuttavia il Papa ha fatto notare ai giovani che pur essendo importanti non devono dimenticare né il loro ambiente né la loro famiglia: «Parla con i tuoi genitori e con i tuoi nonni. Sono la saggezza di ogni popolo. Se volete essere la speranza del popolo, dovete avere memoria, sapere da dove vengo, chi sono e da chi ho ricevuto tutto ciò». ${ }^{4}$

Dalla finestra dell'Arcivescovado in via Franciszkańska il Papa ha mostrato ai giovani la pienezza della vita: gioventù non vuol dire solo la gioia, ma anche i doveri, la morte, la sofferenza. Ha aggiunto anche che nella vita non troveranno risposte a tutte le domande e che dovranno affrontare molti misteri. Ecco perché bisogna essere umili nella vita. Ha fatto loro vedere la vita in tutta la sua complessività. Senza omettere perfino i problemi della vita coniugale, spesso ignorati dalle giovani generazioni. La vita non è una cosa pronta, finita. Bisogna imparare a vivere. Che cosa risulta necessario per questo studio? Il crescere del cuore. In questa arte un giovane non può essere lasciato solo. La pastorale giovanile, una volta creata, deve essere continuamente aggiornata. Perché? Perché cambia l’anima delle giovani generazioni. Ed essa comè? Chi sono i giovani d'oggi?

Pongo questa domanda affinché con l'enfasi delle GMG non venga elusa la sorveglianza pastorale. A Cracovia sono venuti dei giovani buoni e credenti, lélite della Chiesa giovanile. E invece gli altri? Che fine fanno?

\section{Chi sono i giovani polacchi}

Parlando della gioventù e delle sue ambizioni bisogna tener conto che abbiamo a che fare con un gruppo assai eterogeneo e per poter dire qualcosa sui giovani conviene guardare bene tale diversità. I ricercatori della società polacca IQS, specializzata nel predisporre periodicamente rapporti sulla condizione dei giovani in Polonia, hanno suddiviso questo gruppo in cinque così dette tribù. ${ }^{5}$

\footnotetext{
${ }^{4}$ Franciszek Ojciec Święty, Błogosławieni miłosierni, pp. 80-82.

${ }^{5}$ Świat Młodych 3 (2013); il rapporto predisposto dalla agenzia specializzata Grupa IQS di Varsavia, responsabile progetto Anna Maria Szutowicz http://www.raportybadawcze.pl/index. php?option=com_content\&view=article\&id=27:wiat-m-odych-3\&catid=2:raporty (18.01.2017).
} 
La più numerosa (ca. 30\%) viene chiamata Soci. Per loro quello che conta di più è il fashion look. Passano il loro tempo libero nei centri commerciali e su Instagram per essere al corrente di come ci si veste. Amano spendere e gli vanno a genio le feste. Dicono: «Abbiamo il diritto al piacere e alla vita piacevole». Allo stesso tempo più spesso rispetto agli altri temono di non farcela e hanno paura del futuro.

Il secondo gruppo sono i così detti 'casa e chiesa' (23\%). Tradizionalisti e conservatori, sono alla ricerca di ordine e stabilità. Sostengono che la promessa matrimoniale sia indissolubile. I valori fondamentali per loro sono: amore, relazioni, famiglia e amici. Una cosa per loro fondamentale è di essere importanti per gli altri. Sono felici di poter stare più a lungo possibile nella loro casa di famiglia, con un divano consumato, disposti perfino a partecipare alle spese dei genitori.

La terza tribù (19\%) sono i così detti Lifehacker (in altre parole 'pseudo hipster'). Spesso single e con un'altissima autostima. Ottimisti e molto efficaci e pieni d'iniziativa. Credono nella carriera. Spendono soldi per se stessi, trattando se stessi come un marchio e gestendo perfettamente la propria immagine. Osservando i loro profili su Facebook o meglio su Instagram si ha l'impressione di aver a che fare con straricchi e figli di papà. Pubblicano le foto dei viaggi all'estero, ristoranti rinomati ecc. Ma quando entri a casa loro vi trovi un semplice e per niente raffinato set armadio-libreria. Tutto ciò per dare la sensazione di vivere una vita migliore di quella reale.

Il quarto gruppo è costituito dai 'Giovani arrabbiati' (16\%). Spesso sono poveri lavoratori. Il loro slogan è «Polonia allo sfascio». Nel proprio modo di parlare si dimostrano come quelli 'contro il sistema' e 'contro le multinazionali'. Evidentemente risultano arrabbiati. Metà di loro è convinta di dover affrontare un futuro peggiore di quello dei loro genitori. Quasi il 70\% teme per l'indomani, mentre il $60 \%$ dice di non potersi permettere le cose fondamentali. Entrare nel mondo degli adulti risulta per loro estremamente doloroso.

La quinta tribù sono gli Hipster. È la meno numerosa di tutte (12\%), ma sempre presente nei massmedia. Sono quelli della 'Varsavia da bere', autentici figli di papà, ricchi di famiglia. Siccome ricevono dai genitori molti più soldi rispetto agli altri giovani, con estrema facilità dichiarano che il denaro non conta niente, mentre considerano davvero fondamentali creatività e talento. Si sentono i cittadini del mondo. Vogliono esplorare e scoprire. È l'unico gruppo che non dimostri idee nazionalistiche né inclinazioni patriotiche. Risultano più tolleranti. Gli piace giocare e sperimentare con il corpo e con lo stile. Disprezzano 
le griffe più rinomate considerandole come simbolo di ordinarietà e mancanza di individualismo.

\section{L'anima della giovane generazione}

Parlando della gioventù spesso ci avvaliamo di varie ricerche sociologiche, osservazioni, analisi e opinioni. Invece di recente in Polonia sono stati pubblicati due libri di giovani autori che parlano dei loro coetanei. Il primo è intitolato Lemmings - giovani, istruiti, abitanti delle grandi citta ${ }^{6}$ redatto da Jerzy Krakowski. Il secondo è Fare l'erba là dove i cervi bramiscono ${ }^{7}$ di Agnieszka Urbanowska, dove i giovani parlano di se stessi. Quindi vale la pena di guardare bene l'anima delle giovani generazioni che loro stessi svelano davanti a noi.

Lemmings - così si chiamano i giovani, gente ben istruita, proveniente dalla provincia e che vive nei grandi centri urbani. Proprio coloro che hanno lasciato piccole città o villaggi, si sono laureati e hanno trovato lavoro in grosse multinazionali. Multi è il loro ambiente di lavoro. Ma multi ha il proprio stile, il proprio linguaggio e il proprio modo di essere. Chi ottiene il lavoro alla multi deve adeguarsi a tutto ciò. Precisano che in un mondo di valori vivono sul polo opposto, rappresentando la Polonia colorata e gioiosa. Si distaccano dalla tradizione. Il loro luogo preferito d'incontro e di confronto è Starbucks. È di moda essere 'vega' ed 'eco'. Tuttavia, dopo un fine settimana passato dai propri parenti, portano a casa le cottolette e altre delizie della mamma in barattoli ${ }^{8}$ e di nascosto dai colleghi della multi gustano una vita normale. Hanno le loro opinioni circa il patriottismo e la religiosità. Generalmente parlando 'la polonicità è una tamarrata, mentre sulla Chiesa dicono: «Dobbiamo difendere coloro che sono discriminati dal churching. La Chiesa dispone delle proprie regole che però vanno adeguate ai trend di oggi per poter affrontare le sfide del mondo moderno». Alla domanda: «Perché?» rispondono: «Perché noi lo vogliamo». Ovviamente il giorno migliore per fare shopping è domenica. Contestano così il churching arretrato e frequentano centri commerciali più

${ }^{6}$ J. A. Krakowski, Lemingi. Młodzi, wykształceni i z wielkich ośrodków, Warszawa 2014; in polacco il termine «Leming» viene usato nel senso peggiorativo per definire coloro che si identificano con le idee politiche del «centro» rappresentate dal partito Platforma Obywatelska (Piattaforma Civile).

7 A. Urbanowska, Palac trawe na rykowisku, Warszawa 2015.

${ }^{8}$ infatti per questo in Polonia vengono addirittura chiamati „słoiki” (barottoli). 
en vogue, in quanto - come dicono - bisogna farla finita con la tradizione di andare in chiesa la domenica.

La filosofia dei lemmings viene perfettamente illustrata dalla discussione sul Natale. Loro dicono così: «Visto che l'Occidente festeggia Winter Holiday, noi vogliamo festeggiare la Pausa Umanistica e Culturale». Al posto di una tradizionale vigilia di Natale ${ }^{9}$ propongono una vigilia umanistica. Sostituiscono un rituale cenone polacco con un pranzo in stile vegetariano. Consapevolmente rinunciano al tavolo, quale arredo che rappresenta la comunità familiare, e mangiano seduti sul pavimento. «Dato che ci discostiamo dai paradigmi tradizionali - afferma uno di loro - lasciamo perdere lostia. ${ }^{10}$ Liberiamoci dunque dalla schiavitù dell'ostia e gustiamo germogli e sushi». Qualcuno ha osato proporre: «Almeno facciamoci gli auguri». «Ma non è per niente trendy augurarsi 'tante buone cose'», ha risposto un altro. "Il bene e il male sono categorie appartenenti al churching da cui vogliamo scappare. Forse è meglio augurarsi 'tante cose sfiziose', quindi ognuno lo potrà adeguare alle proprie esigenze». Nessun albero di natale, né mormorare canti natalizi - bisogna finalmente smettere con questi costumi provinciali. Il Winter Holiday viene passato in palestra. È così importante stare in forma!

Invece Fare l'erba là dove i cervi bramiscono è una storia sui giovani cattolici in un mondo postmoderno. Una ragazza 26enne, figlia di un rinomato critico letterario e storico della letteratura, cerca di mostrare nel suo libro un tentativo di conciliare l'edonismo con la fede. Non si tratta di respingere la fede, ma di trovare un compromesso. Godersi il mondo e salvare l'anima. Gaja - la protagonista del romanzo - rappresentante della generazione GPII, single, si occupa di arti applicate. È veramente affascinata dalla cultura pop dell'epoca post moderna. Immersa nell'euforia di Internet e nel mondo delle emozioni virtuali. Si dichiara cattolica. Di tanto in tanto frequenta l'ambiente domenicano di Cracovia. Lo considera come un relitto, un ricordo, un qualcosa di nostalgico. Pur dichiarandosi come una persona credente, non ha scrupoli nel convivere con il suo ragazzo, docente universitario, bisessuale ed edonista che si droga e non evita l'alcol. La ragazza sta perdendo l'anima ma ai propri occhi non si sente cattiva in quanto sostiene sua sorella abbandonata dal marito. Per lei il sesso è una sua scelta personale. La religione invece è una specie di sentimento

\footnotetext{
9 In Polonia il punto centrale delle feste Natalizie è proprio la cena solenne del 24 dicembre.

${ }^{10}$ Una delle principali tradizioni natalizie consiste nel dividersi l'ostia facendosi gli auguri durante il cenone del 24 dicembre.
} 
per i bei tempi dell'infanzia. Quando nello schianto dell'aereo presidenziale a Smoleńsk muore il suo primo amore del liceo, si risveglia. La devastazione spirituale provocata dal mondo è enorme. Nonostante tutto cerca di convertirsi. Ma questa conversione è nient'altro che un tentativo di conciliare due mondi: quello moderno e quello cattolico. Deliziosamente spudorata nel suo peccare, cerca di salvare quei residui di cattolicesimo e la sua coesione spirituale. Sembra comunque difficile trovare una buona soluzione che permetterebbe di conciliare il cristianesimo con la distruggente modernità. Risulta problematico concordare l'edonismo con la fede profonda.

Ecco il dilemma dei giovani...

Forse oggi è anche il dilemma della Chiesa?!?!

\section{I partecipanti delle GMG - élite della chiesa}

In occasione delle GMG le ricerche sui giovani sono state svolte dall' Istituto di Statistica della Chiesa Cattolica. I dati pubblicati rivelano che tra varie aspettative riguardanti le GMG quella predominante in modo assoluto era il desiderio di esperienze di natura religiosa. Al primo posto è stata indicata la speranza di sperimentare la presenza di Dio $(68,5 \%)$, al secondo la voglia di vedere e sentire Papa Francesco (55\%). ${ }^{11}$

Tra gli attesi benefici della partecipazione alle GMG, in primo luogo i giovani hanno indicato i vantaggi di carattere religioso. Ben 68,4\% degli intervistati si augurava di avere - grazie alle GMG - un rapporto più stretto con Dio. ${ }^{12}$

Tra le principali celebrazioni religiose svoltesi durante le GMG quelle più toccanti sono state la Messa conclusiva (durante la quale la metà degli intervistati dichiara di aver sperimentato la presenza di Dio) nonché la Via Crucis in Błonia (durante la quale il 40,3\% dei presenti ha sperimentato Dio). Invece per quanto riguarda la crescita spirituale l'evento più importante risulta la Messa finale. ${ }^{13}$

${ }^{11}$ Raport $z$ badania odbioru uczestników Światowych Dni Młodzieży Kraków 2016 i ich kulturowych kontekstów predisposto dall'Istituto di Statistica della Chiesa Cattolica, disponibile sul sito: http://www.nck.pl/badania/318392-raport-z-badania-odbioru-swiatowych-dni-mlodziezy-krakow-2016-i-ich-kulturowych-kontekstow/ (18.01.2017).

12 Raport z badania odbioru uczestników Światowych Dni Młodzieży Kraków 2016 i ich kulturowych kontekstów.

${ }^{13}$ Raport z badania odbioru uczestników Światowych Dni Młodzieży Kraków 2016 i ich kulturowych kontekstów. 
La partecipazione alle GMG ha causato nei giovani una maggiore approvazione dell'autorevolezza della Chiesa, ma anche ha suscitato in loro la convinzione sull'autenticità del cattolicesimo. In fine molto più forte risulta perfino la loro identificazione con l'identità cattolica.

Per la maggioranza dei giovani la partecipazione alle GMG risulta una delle migliori esperienze della loro vita $(47,3 \%)$ o almeno unesperienza molto positiva $(39,3 \%) .{ }^{14}$

In seguito a queste ricerche emerge che per molti giovani le GMG sono state unopportunità per impegnarsi nel volontariato. È senza dubbio qualcosa di positivo. Tuttavia ci sono alcuni punti interrogativi:

1. fino a che punto si trattava di una rivolta spontanea, di un attivismo a breve durata e fino a che punto esso sarà un impegno più profondo, più consapevole e duraturo?

2. quale era il loro punto di riferimento: la motivazione orizzontale (lo faccio per me stesso) o quella verticale (lo faccio per amore per Dio)?

La risposta viene fornita dagli stessi giovani che come motivo della loro partecipazione al volontariato indicano:

1. sviluppo intellettuale,

2. capacità linguistiche e comunicative,

3. voglia di stabilire relazioni con altra gente.

Le analisi condotte tra i partecipanti alle GMG dimostrano che i giovani si attivano tramite varie comunità e movimenti ecclesiastici e non a livello della parrocchia nel suo insieme. Tra i movimenti più diffusi occorre menzionare: Oasi (Luce e vita) e tutte le comunità operanti sotto forma di Scuola per la Nuova Evangelizzazione.

Secondo i giovani cattolici quelli che più influenzano i loro atteggiamenti sono:

- un buon prete,

- una comunità religiosa,

- i ritiri spirituali.

Come risulta dalle analisi sulla gioventù e sulla sua religiosità qui sopra presentate, in linea generale possiamo dire che sta crescendo una generazione di consumatori. Aumenta anche il divario tra la fede dichiarata, le pratiche

\footnotetext{
${ }^{14}$ Raport $z$ badania odbioru uczestników Światowych Dni Młodzieży Kraków 2016 i ich kulturowych kontekstów.
} 
religiose e la morale. Fondamentalmente i giovani non si interessano della fede. In più hanno una visione negativa della parrocchia e dei preti.

Tuttavia il gruppo che ha partecipato alle GMG costituisce l'élite della Chiesa. Questi giovani vivono la loro fede con coraggio e consapevolezza. Si rendono conto di essere diversi. Cè in loro la voglia e il bisogno di condividere la loro fede e di stabilire relazioni. Giudicano male la religiosità dei propri nonni ma anche dei genitori, trovando la loro fede troppo 'ritualistica' e incoerente. ${ }^{15}$

Mentre loro vogliono univocità. Costituiscono una nuova qualità della fede. Non sono numerosi. Ma forti. Si incuneano in un ambiente sempre più secolarizzato.

\section{I suggerimenti per la pastorale giovanile}

Le GMG hanno accumulato un notevole capitale di giovani. E ora si chiedono: Che facciamo adesso?

Quindi, quali sono le indicazioni per la pastorale giovanile dopo questo grande evento? Vorrei sottolineare quattro idee principali.

Primo: Papa Francesco ha detto: «Dove cè Cristo, cè una festa della fede». Per questa festa della fede si intende probabilmente la Santa Messa. Qualora la pastorale dovesse svelare ai giovani il mondo di Dio, qualora dovesse aiutarli a incontrare Gesù, bisogna di sicuro riflettere sulla questione delle Messe domenicali per i giovani. Sappiamo che la fede non nasce dalla catechesi. Quest'ultima costituisce soltanto uno spettro della conoscenza di Dio che può servire come sostegno alla fede. Dio viene incontro all'uomo nell'Eucarestia e nei sacramenti. La Messa per i giovani nelle parrocchie si limita spesso a quei giovani che si stanno preparando alla Cresima. Ma è soltanto una piccola parte dei giovani residenti in una data parrocchia. I giovani credenti devono avere una vera e propria festa della fede, che è l'incontro con Cristo. Oggi, dopo le GMG, possiamo affermare con certezza che il modo in cui la preghiera viene vissuta e preparata è un po' diverso da quello a cui ci siamo abituati nelle parrocchie.

Come quindi organizzare la festa della fede per i giovani? Forse conviene parlarne con loro? In ogni caso bisogna fare qualcosa.

15 O młodzieży po Światowych Dniach Młodzieży, l'intervista al vescovo A. Wodarczyk, „Homo Dei” (2016) nr 4, pp. 107-115. 
La seconda indicazione riguarda coloro che erano direttamente coinvolti nelle GMG. Si tratta di volontari, membri di vari movimenti e comunità; questi costituiscono un grande potenziale. Forse proprio loro potrebbero diventare un vero inizio per un rinnovamento della pastorale giovanile nelle parrocchie? Ma non si tratta soltanto dello 'sfruttamento' ecclesiale di questa gente. Loro hanno bisogno di approfondire la propria vita spirituale, hanno bisogno della formazione. È per loro che bisogna prevedere una forma della pastorale che potrebbe sviluppare la loro attività ecclesiale. Sono molto coinvolti e pieni di buone intenzioni. Lasciati senza alcuna assistenza potrebbero diventare dei 'parrocchiali frustrati'.

La terza indicazione riguarda quei giovani che non fanno parte in alcun modo della pastorale giovanile. Alla Pontificia Facoltà di Teologia di Varsavia negli ultimi due anni, proprio nell'ottica delle GMG in Polonia, si era svolto il corso intitolato Studium di volontariato che ha visto la partecipazione di circa quattrocento persone. La maggior parte di loro apparteneva ai vari movimenti e/o comunità. Un centinaio di essi non proveniva però da alcuna organizzazione ecclesiastica. Ciò dimostra che oltre ai movimenti quali Oasi, Rinnovamento carismatico cattolico e Cammino neocatecumenale cè ancora bisogno all'interno della Chiesa di unaltra formula per i giovani. E questo può essere ritenuto lo spazio per espandere una nuova forma della pastorale giovanile. Le ricerche svolte tra i giovani mostrano che essi iniziano a diventare sazi del consumismo. Dicono apertamente: «Basta succhiare a vuoto! Abbiamo bisogno di una vita più piena di significato». Queste affermazioni dovrebbero essere interessanti per la Chiesa.

La quarta e ultima indicazione riguarda noi stessi: pastori e catechisti. I giovani si aspettano da noi la freschezza, l'entusiasmo e la testimonianza. Come farlo? Mi permetto di condividere con voi una mia esperienza personale...

Lo scorso Natale avevo battezzato nella mia parrocchia di provenienza il figlio del mio figlioccio. Un grande evento, l'atmosfera di festa, tutta la famiglia riunita: un'esperienza stupenda. Dopo la Messa siamo tornati a casa a mangiare, un bel pranzo di Natale, auguri, chiacchiere. Ad un certo momento mi sono avvicinato alla culla per rivolgermi al bambino. Suo padre ha alzato il piccolo dal lettino dicendomi: «Zio, dai, prendilo!». Intimidito ho chiesto: «Ma come?». Sembrava così piccolo e fragile, non sapevo come fare. Il mio figlioccio disse: «Zio, dai, lo hai appena battezzato quindi non avere paura!». Ho preso il bambino in braccio e l'ho stretto delicatamente al mio petto. Lui si è attaccato a me con tutto il suo piccolo corpicino e all'improvviso entrambi ci siamo sentiti sicuri. 
Dopo un attimo ho sentito battere il suo cuore. Siamo rimasti così per un bel po'. La sera sono tornato a Varsavia. Quell'incontro con il piccolo non mi lasciava in pace. Ho pensato: «Ogni giorno celebro l'Eucarestia, tengo in mano Gesù. Ma sento il battere del Suo cuore? So stringerLo a me? Probabilmente è più facile tenerLo a distanza. Come mai? Perché così non devo prendermi cura di Lui. Una cosa è avvicinarsi al lettino e parlare al bambino, un'altra prenderlo in braccio. Quando prendiamo in braccio il bambino bisogna prendersi cura di lui. Dargli una sensazione di sicurezza. Lo stesso vale con Dio. È più facile andare alla Messa, dire le preghiere e poi occuparsi dei propri affari. Ma prendere Dio dentro il proprio cuore, occuparsi di Lui, prendersi cura di Lui è molto più difficile, perché significa iniziare a creare una relazione, un legame».

Vicinanza e cordialità. Penso che abbiamo difficoltà con la prossimità di Gesù. Come prenderLo in braccio e stringerLo al cuore? Come sentire che questo Gesù, presente nell'Eucarestia, mi sta sempre vicino nella mia quotidianità? Ma forse cercare la vicinanza di Dio nella quotidianità ci aiuta a vedere in modo diverso uno studente durante la lezione di catechismo? Lo insegno prendendomi cura di Dio che cè in lui. A volte questo prendersi cura sará faticoso, come faticose sono tutte le cure. Una madre che ama i suoi figli si sacrifica per loro, cerca il modo migliore per prendersi cura di loro, a volte ciò risulta difficile, ma lei non smette mai di cercare. Perché? Perché ama.

Che cosa attendono i giovani dal catechista, dal prete? Amore e passione.

\section{Bibliography}

Franciszek Ojciec Święty, Błogosławieni miłosierni. Słowa do młodych, Kraków 2016. Krakowski J. A., Lemingi. Młodzi, wykształceni i z wielkich ośrodków, Warszawa 2014.

O młodzieży po Światowych Dniach Młodzieży, l'intervista al vescovo A. Wodarczyk, „Homo Dei” (2016) nr 4, pp. 107-115.

Raport z badania odbioru uczestników Światowych Dni Młodzieży Kraków 2016 i ich kulturowych kontekstów predisposto dall'Istituto di Statistica della Chiesa Cattolica, http://www.nck.pl/badania/318392-raport-z-badania-odbioru-swiatowych-dnimlodziezy-krakow-2016-i-ich-kulturowych-kontekstow/ (18.01.2017).

Świat Młodych 3 (2013); il rapporto predisposto dalla agenzia specializzata Grupa IQS di Varsavia, responsabile progetto Anna Maria Szutowicz http://www.raportybadawcze.pl/ index.php?option=com_content\&view=article\&id=27:wiat-m-odych-3\&catid=2:raporty (18.01.2017).

Urbanowska A., Paląc trawę na rykowisku, Warszawa 2015. 